OPEN ACCESS

Edited by:

Klaus Libertus,

University of Pittsburgh, USA

Reviewed by:

Amy Needham,

Vanderbilt University, USA

Daniel Casasanto,

The University of Chicago, USA

${ }^{*}$ Correspondence:

George F. Michel

gfmichel@uncg.edu

Specialty section:

This article was submitted to

Developmental Psychology,

a section of the journal

Frontiers in Psychology

Received: 17 December 2015 Accepted: 07 March 2016

Published: 23 March 2016

Citation:

Michel GF, Campbell JM

Marcinowski EC, Nelson EL and Babik I (2016) Infant Hand Preference and the Development of Cognitive Abilities.

Front. Psychol. 7:410

doi: 10.3389/fpsyg.2016.00410

\section{Infant Hand Preference and the Development of Cognitive Abilities}

\author{
George F. Michel'1*, Julie M. Campbell' ${ }^{1}$ Emily C. Marcinowski², Eliza L. Nelson ${ }^{3}$ and \\ Iryna Babik ${ }^{4}$
}

'Department of Psychology, The University of North Carolina at Greensboro, Greensboro, NC, USA, ${ }^{2}$ Department of Physical Therapy, Virginia Commonwealth University, Richmond, VA, USA, ${ }^{3}$ Department of Psychology, Florida International University, Miami, FL, USA, ${ }^{4}$ Department of Physical Therapy, University of Delaware, Newark, NJ, USA

Hand preference develops in the first two postnatal years with nearly half of infants exhibiting a consistent early preference for acquiring objects. Others exhibit a more variable developmental trajectory but by the end of their second postnatal year, most exhibit a consistent hand preference for role-differentiated bimanual manipulation. According to some forms of embodiment theory, these differences in hand use patterns should influence the way children interact with their environments, which, in turn, should affect the structure and function of brain development. Such early differences in brain development should result in different trajectories of psychological development. We present evidence that children with consistent early hand preferences exhibit advanced patterns of cognitive development as compared to children who develop a hand preference later. Differences in the developmental trajectory of hand preference are predictive of developmental differences in language, object management skills, and tool-use skills. As predicted by Casasanto's body-specificity hypothesis, infants with different hand preferences proceed along different developmental pathways of cognitive functioning.

Keywords: hand preference, infants, embodied cognition, cognitive development

\section{INTRODUCTION}

For the last four decades, one of us (GFM) has been investigating the factors affecting the development of infant hand-use preferences as a means of understanding how infant sensorimotor experiences contribute to the development of hemispheric differences in language processing (cf., Michel, 1988, 1991). The theoretical foundation of this work was derived, in part, from theories of Piaget (1952) and Bruner (1973), which proposed that infant sensorimotor experiences formed the foundation of symbol formation, language skill, concept formation, and reasoning. Also, developmental psychobiological evidence about how nervous system functioning could be shaped by early experience was used to support the notion that infant hand preferences could shape functional lateralization of the hemispheres (Michel, 1998, 2002). Since most neuropsychological research was, and is, conducted within the framework that hemispheric specialization of function derives from gene controlled differences in the structural organization of the two hemispheres, hand preference and related cognitive functions are often considered to be derivative of hemispheric specialization rather than to be contributors to it. However, in 
the last decade, some forms of embodiment theory (e.g., Barsalou, 2008; Casasanto, 2009) have reopened theoretical consideration that sensorimotor experiences can contribute to the functional organization of the brain.

Certain forms of embodiment theory (e.g., Barsalou, 2008; Casasanto, 2009, 2011) propose that the development of symbolic cognitive and social knowledge depends on the individual's sensorimotor engagement with social companions and physical objects during infancy. Infant vocalizations, facial expressions, and body postures (as sensorimotor actions) elicit actions from social companions, which provide the developing infant with information about the rules of social-relational engagement. This is similar to how the manipulation of objects reveals object properties, their relations, and rules of combination. How infants engage with these aspects of their environment sculpts their brain functioning and structure (Boulenger et al., 2009), which, in turn, affects their cognitive and social development. Thus, if there are group differences in such patterns of engagement, then there ought to be group differences in cognitive and social abilities. One way of testing whether such engagements with objects shape cognitive development would be to compare the cognitive development of groups of individuals who engage the world differently during infancy.

We propose that the development of infant hand preferences creates groups of infants who engage the world differently and hence, they should develop differences in cognitive functioning. This thesis is in accordance with Casasanto's (2011) bodyspecificity hypothesis and the thesis of this Frontiers' special issue (contributions of sensorimotor experience to cognitive development). We briefly review some of our studies which demonstrate that consistent infant hand preferences predict developmental advances in language development, tool-use, and objects construction skill. We propose that these advances not only contribute to the individual's development of language, concept formation, and reasoning, but also the individual's functional organization of the brain.

\section{THE HAND PREFERENCE PHENOMENON}

Hand preferences in adults are related to differences in hemispheric specialization for language skills (e.g., Corballis, 2009; Häberling et al., 2015), word processing differences between hemispheres (e.g., Willems et al., 2010), and a remarkably wide range of performance differences on tasks of cognitive, social, and emotional functioning (Annett, 2002). Moreover, apparently atypical hand preference development seems to be related to nearly every mental and medical health issue (e.g., Michel et al., 2013b, pp. 207-208). Consequently, some investigators have argued that examination of the cognitive and social abilities of different hand preference groups is the perfect test for evaluating embodiment theory (e.g., Casasanto, 2009). Hand preference represents different patterns of hemispheric specialization and such specialization may be relevant for the manifestation of specific aspects of cognitive, social, and emotional functioning.
Therefore, the development of hand preference ought to relate to the typical and atypical development of many psychological functions.

However, before we can understand how hand preferences could contribute to variability in embodied cognitive experiences, we first must understand how hand preferences develop. Hand preference is the product of multifaceted developmental processes that begin before birth and expand during early infancy (Michel et al., 2013b). We have found that hand preferences are developing in a cascading fashion with preferences for earlier developing manual skills (e.g., reaching, grasping/acquisition) concatenating into preferences for later developing skills (e.g., unimanual and bimanual manipulation, artifact construction, and tool-use). We observed that a hand preference for acquiring objects starts manifesting before the age of 6 months, becomes prominent during 6-12 month period, and declines thereafter (Michel, 2002; Ferre et al., 2010; Michel et al., 2014). Also, although unimanual manipulation skills develop by 7-8 months, only by $10-11$ months of age do infants exhibit a hand preference for unimanual manipulation and that preference matches the preference for acquisition (Campbell et al., 2015a). By 13-14 months of age, there is a significant increase in hand preferences for role-differentiated bimanual manipulation (RDBM, Babik and Michel, 2016). The hand preference for RDBM seems to stabilize by the age of 18 months (Nelson et al., 2013) with $80 \%$ of toddlers maintaining the same preference to 24 months. A consistent hand preference for RDBM likely influences the development of hand preference for tool-use, since $\mathrm{RDBM}$ is an object manipulation pattern characteristic of most actions involved in tool construction and use throughout the life-span (Vauclair, 1984). Although a right hand preference predominates in all infant manual skills, the hand preferences appear to be distributed continuously across infants (similarly to adult hand preferences, Annett, 2002).

Although approximately $12 \%$ of infants have a consistent left hand preference (Michel et al., 2014), the left hand preference does not appear to be as robust as the right preference. In part, this may be a consequence of a maternal influence on the development of infant hand preferences (Harkins and Michel, 1988; Michel, 1992, 1998; Mundale, 1992, unpublished). Righthanded mothers unintentionally engage the use of their infant's right hand during object play (Michel, 1998). In contrast, although left-handed mothers use their left hand more when playing with their infants, the difference in their hand use is small compared to the overwhelming use of the right hand by righthanded mothers. Thus, left preference infants of right-handed mothers (the majority of left preferring infants) are likely to be encouraged (by their mother's actions during socially interactive object play) to use their right hand much more than right preference infants of left-handed mothers (a minority of right preferring infants) are encouraged to use their left hand. Indeed, infants initially manifesting left-hand preference for acquiring objects who had right-hand preferring mothers significantly reduced their left-hand preference by 11 months; whereas infants initially manifesting right-hand preference who had right-hand preferring mothers strengthened their right-hand preference by 11 months (Michel, 1992). 


\section{ASSESSING HAND PREFERENCE DEVELOPMENT USING A TRAJECTORY BASED ANALYSIS}

The expression of infant hand preferences reflects the consequences of an immature but rapidly developing nervous system and expression can vary according to such factors as circadian rhythm, situational arousal, and the development of other neuromotor abilities, such as postural control and locomotion (Corbetta and Thelen, 2002; Babik et al., 2014). Moreover, the identification of a preference appears to be sensitive to various assessment procedures and conditions (Campbell et al., 2015b). Therefore, assessment of infant hand preferences requires longitudinal designs using tasks that are relatively similar, across age, in the manual challenge that they present for the infant. Object acquisition skills can be used to assess the development of hand preferences during the period from 6 to 14 months of age because this manual skill is prevalent in the infant's repertoire but it is sufficiently challenging to elicit a hand preference across this age period. Moreover, it is incorporated into all other manual skills involving object manipulation (construction of objects and tool use). A hand preference in reaching and object contact predicts the hand preference in object acquisition (Michel and Harkins, 1986) and the hand preference for acquiring objects predicts the hand preference for unimanual object manipulation (Hinojosa et al., 2003; Campbell et al., 2015a) and RDBM (Nelson et al., 2013; Babik and Michel, 2016). Thus, early-developing hand preference for object acquisition is pivotal for the development of hand preference for other more sophisticated manual skills.

We hypothesized that there ought to be consistent developmental trajectories for object acquisition hand preference despite some variation across assessment ages. Nine monthly assessments during the 6-14 month period permitted reliable identification of four latent groups according to their pattern of developmental trajectories using group based trajectory modeling (GBTM, Nagin, 2005; Michel et al., 2013a). GBTM permits identification distinctive patterns in the distribution of a sample's trajectories to define the infant's hand preference We found that $32 \%$ of 380 infants have consistent right-hand preferences from 6 to 14 months of age, 12\% have consistent left preferences, and $26 \%$ have a developmental trajectory trending toward a later developing right preference. The remaining $30 \%$ of infants had a consistent trajectory of hand use that showed no differences in hand-use across the ages. Hierarchical Linear Modeling (HLM, Raudenbush et al., 2004), confirmed that the infants assigned to these four latent classes exhibited significantly different trajectories in their development of hand preferences. Infants with a right preference have established that preference by 6 months of age and maintain it for the next 9 months (with a slight decrease in right hand use by 13 and 14 months). Infants with a left preference had not established that preference before 8 months of age, but maintain it thereafter. Infants with a trend toward a right preference start at 6 months without a preference but have established a right preference by 14 months. Those infants without a hand preference maintain that throughout the 6-14 month period. Thus, by the beginning of the second year, hand preferences are exhibiting the common character of a right preference for most and a left preference for about $12 \%$ of the infants and about 30\% with unclear preferences. Of course, the number of groups identified is less important than the recognition that it is only by the collection of such longitudinal data that consistencies across assessment periods can be identified. It is those consistencies of preference that reflect the operation of neural mechanisms that ought to contribute to the development of other mechanisms associated with cognitive functioning.

\section{HAND PREFERENCE AND LANGUAGE}

The development of manual skills dynamically shifts the way infants experience their world, and various changes in motor skills have been linked to changes in language ability (e.g., Iverson, 2010). Here, we highlight our longitudinal studies that have used a trajectory-based approach to characterize hand preference and address how hand preference trajectories may be differentially related to language acquisition.

Nelson et al. (2014) hypothesized that a consistent infant hand preference was a marker for advanced object manipulation skill, whereas an inconsistent preference would be an indicator of a lower skill level and perhaps a different pattern of hemispheric specialization. Nelson et al. (2014) described trajectories in the timing and direction of hand preference among children assessed monthly as infants (6-14 months) and then as toddlers (1824 months): children with consistent right-hand preference as infants who remained right-handed as toddlers, and children without consistent hand preference as infants who became either right-handed or left-handed as toddlers. Consistency versus inconsistency of hand preference from infancy through toddlerhood explained $25 \%$ of the variance in language ability at 2 years of age. Also, consistent right-hand preference from infancy was associated with advanced language skills. Gonzalez et al. (2015) extended this work to include language outcome at 3 years in the same sample and found that children with a consistent hand preference trajectory as toddlers had higher expressive language scores. Thus, early, consistent hand preferences may facilitate the development of language (Nelson et al., 2014; Gonzalez et al., 2015). Although more works needs to be done, Michel et al. (2013a) used Arbib's schema theory (Arbib, 2006) to delineate some of the mechanisms by which the sensorimotor experience associated with a hand preference could contribute to the neural control of expressive language skills.

\section{HAND PREFERENCE AND THE MANUAL CONTROL OF OBJECTS}

It is reasonable to assume that infants with a hand preference for engaging with objects would develop greater manual skill and proficiency with the preferred hand and that preference would affect the development of their manual control of objects. Object 
construction requires manually merging multiple objects into a single, unifying structure, such as stacking blocks into a tower or assembling a puzzle (Marcinowski, 2015). Object construction has recently been related to a variety of cognitive skills at later ages, including mathematical ability (Wolfgang et al., 2003; Nath and Szucs, 2014), language (Marcinowski and Campbell, 2015) and visuospatial skill (Caldera et al., 1999; Levine et al., 2011; Verdine et al., 2014).

Marcinowski (2015) found that infants with a consistent hand preference develop stacking more quickly during 10-14 months period than infants without a hand preference. Consistent leftand right-preferring infants manifested greater stacking skill at 14 months, than infants without a consistent hand preference. Moreover, infants with a trending right preference did not differ in the development of their stacking skill from infants without a preference. Since the trending right group did not exhibit a hand preference for acquisition during the 6-9 months period before stacking began to be assessed, they likely had not developed the manual proficiency needed to stack objects (Chen et al., 2010). Thus, the consistency of a hand preference changes the relation between a hand preference and the cognitive skill of object construction.

Also, Kotwica et al. (2008) reported that infants with consistent hand preferences across four assessment periods (at $7,9,11$, and 13 months of age) are more effective with the object management skill of object storage than infants without a consistent preference during that period. When infants are given multiple toys (one at a time), they must develop the ability to manipulate and manage these objects so that the latter are available for future interaction. Infants with consistent hand preference demonstrated a greater skill for object storage, such as placing objects in reachable locations and intermanual transfer, than infants without a hand preference (Kotwica et al., 2008). By storing objects more effectively, infants with a hand preference can explore properties of objects, understand relations between objects, and "plan" actions more effectively than infants without a preference (cf., Bruner, 1973). Indeed, Bruner (1973) considered object storage skills to be important for the development of symbolic representation (and hence language development), since an unused, but stored object must be mentally represented by the infant for later retrieval.

Tool use is another important cognitive skill that often involves imitation of complex actions, planning, decisionmaking, and the ability to account for spatial and temporal characteristics of objects, their properties, and the situation. Many have argued that tool use requires advanced symbolic thinking and representational means-end analysis (Bates et al., 1980), advanced causal understanding (Carpenter et al., 1998; Buttelmann et al., 2008), and an achievement of spatial reasoning that permits coordinating multiple mobile frames of reference

\section{REFERENCES}

Annett, M. (2002). Handedness and Brain Asymmetry: The Right Shift Theory. East Sussex: Psychology Press.

Arbib, M. A. (2006). "The mirror-system hypothesis on the linkage of action and language," in Action to Language via the Mirror Neuron
(Lockman, 2000). Fraz et al. (2014) tested the development of the tool use skill longitudinally from 10 to 14 months in 60 infants with right, left, or no hand preference for acquiring objects. They found that infants with consistent right or left hand preference out-performed those without a hand preference in the number of successfully completed tool-using actions at the ages of 10, 11, and 12 months. However, after 12 months differences between the hand preference groups ceased to be statistically significant. Fraz et al. (2014) concluded that early-development of consistent hand-use preferences for acquiring objects facilitates the onset of the cognitive skill of tool use. Thus, we have shown how longitudinal assessments of the consistency of hand preferences relate to the development of the manipulation of objects that are considered to contribute to the development of symbolic cognitive abilities.

\section{CONCLUSION}

We have demonstrated that early-established hand preferences (revealed by their consistency across longitudinal assessments) for object acquisition and manipulation of objects significantly predict developmental advancement of such important elements of cognitive development as expressive language, object construction, object management skills, and tool use. Thus, it is important that longitudinal consistency in infant hand preferences be taken into account while exploring patterns of neurobehavioral functioning and cognitive development. Our results are consistent with the predictions of Casasanto's (2009, 2011) body-specificity hypothesis. Having a more practiced, preferred hand could assist infants in scaffolding their manual proficiency and hence their comprehension of the properties of objects. Such comprehension, in turn, could contribute to the development of other cognitive abilities as revealed in object construction, tool-using, and language development. What remains to be demonstrated is how these differences in hand preference development have influenced the functional organization of the infant's brain.

\section{AUTHOR CONTRIBUTIONS}

JC, EM, EN, IB, and GM conceptualized and wrote the paper.

\section{FUNDING}

Preparation of this manuscript was supported by NSF grant DLS0716045 awarded to GM and NIH T32HD007376 fellowship awarded to JC and EN.

System, ed. M. A. Arbib (Cambridge: Cambridge University Press), $1-45$.

Babik, I., Campbell, J. M., and Michel, G. F. (2014). Postural influences on the development of infant lateralized and symmetrical hand-use. Child Dev. 85, 294-307. doi: 10.1111/cdev. 12121 
Babik, I., and Michel, G. F. (2016). Development of Role-Differentiated Bimanual Manipulation in Infancy: part 2. Hand preferences for object acquisition and RDBM - Continuity or discontinuity? Dev. Psychobiol. 58, 257-267. doi: $10.1002 / \operatorname{dev} .21378$

Barsalou, L. (2008). Grounded cognition. Annu. Rev. Psychol. 59, 617-645. doi: 10.1146/annurev.psych.59.103006.093639

Bates, E., Carlson-Luden, V., and Bretherton, I. (1980). Perceptual aspects of tool using in infancy. Infant Behav. Dev. 3, 127-140. doi: 10.1016/S01636383(80)80017-8

Boulenger, V., Hauk, O., and Pulvermüller, F. (2009). Grasping ideas with the motor system: semantic somatotopy in idiom comprehension. Cereb. Cortex 19, 1905-1914. doi: 10.1093/cercor/bhn217

Bruner, J. S. (1973). Beyond the Information Given. New York, NY: W. W. Norton \& Company.

Buttelmann, D., Carpenter, M., Call, J., and Tomasello, M. (2008). Rational tool use and tool choice in humans and great apes. Child Dev. 79, 609-626. doi: 10.1111/j.1467-8624.2008.01146.x

Caldera, Y. M., Culp, A. M., O’Brien, M., Truglio, R. T., Alvarez, M., and Huston, A. C. (1999). Children's play preferences, construction play with blocks, and visual-spatial skills: are they related? Int. J. Behav. Dev. 23, 855-872. doi: 10.1080/016502599383577

Campbell, J. M., Marcinowski, E. C., Babik, I., and Michel, G. F. (2015a). The influence of a hand preference for acquiring objects on the development of a hand preference for unimanual manipulation from 6 to 14 months. Infant Behav. Dev. 39, 107-117. doi: 10.1016/j.infbeh.2015. 02.013

Campbell, J. M., Marcinowski, E. C., Latta, J., and Michel, G. F. (2015b). Different assessment tasks produce different estimates of handedness stability during the eight to 14 month age period. Infant Behav. Dev. 39, 67-80. doi: 10.1016/j.infbeh.2015.02.003

Carpenter, M., Akhtar, N., and Tomasello, M. (1998). Fourteen-through 18-monthold infants differently imitate intentional and accidental actions. Infant Behav. Dev. 21, 315-330. doi: 10.1016/S0163-6383(98)90009-1

Casasanto, D. (2009). Embodiment of abstract concepts: good and bad in rightand left-handers. J. Exp. Psychol. Gen. 138, 351-367. doi: 10.1037/a0015854

Casasanto, D. (2011). Different bodies, different minds: the body specificity of language and thought. Curr. Dir. Psychol. Sci. 20, 378-383. doi: 10.1177/0963721411422058

Chen, Y.-P., Keen, R., Rosander, K., and von Hofsten, C. (2010). Movement planning reflects skill level and age changes in toddlers. Child Dev. 81, 1846-1858. doi: 10.1111/j.1467-8624.2010.01514.x

Corballis, M. C. (2009). The evolution and genetics of cerebral asymmetry. Philos. Trans. R. Soc. B 364, 867-879. doi: 10.1098/rstb.2008.0232

Corbetta, D., and Thelen, E. (2002). Behavioral fluctuations and the development of manual asymmetries in infancy: contributions of the dynamic systems approach. Handb. Neuropsychol. 8, 311-330.

Ferre, C. L., Babik, I., and Michel, G. F. (2010). Development of infant prehension handedness: a longitudinal analysis during the 6- to 14-month age period. Infant Behav. Dev. 33, 492-502. doi: 10.1016/j.infbeh.2010.06.002

Fraz, F., Babik, I., Varholick, J., and Michel, G. F. (2014). Development of hand-use preference for tool-use in infancy. Dev. Psychobiol. 57:S13.

Gonzalez, S. L., Nelson, E. L., Campbell, J. M., Marcinowski, E. C., Coxe, S., and Michel, G. F. (2015). 18-24 months handedness predicts 36 months expressive language skills. Dev. Psychobiol. 57:S16.

Häberling, I. S., Steinemann, A., and Corballis, M. C. (2015). Cerebral asymmetry for language: comparing production with comprehension. Neuropsychologia 80 , 17-23. doi: 10.1016/j.neuropsychologia.2015.11.002

Harkins, D. A., and Michel, G. F. (1988). Evidence for a maternal influence on infant hand-use preferences. Dev. Psychobiol. 21, 535-542. doi: $10.1002 /$ dev.420210604

Hinojosa, T., Sheu, C.-F., and Michel, G. F. (2003). Infant hand-use preferences for grasping objects contributes to the development of a hand-use preference for manipulating objects. Dev. Psychobiol. 43, 328-334. doi: 10.1002/dev. 10142

Iverson, J. M. (2010). Developing language in a developing body: the relationship between motor development and language development. J. Child Lang. 37, 229-261. doi: 10.1017/S0305000909990432
Kotwica, K. A., Ferre, C. L., and Michel, G. F. (2008). Relation of stable hand-use preferences to the development of skill for managing multiple objects from 7 to 13 months of age. Dev. Psychobiol. 50, 519-529. doi: 10.1002/dev.20311

Lockman, J. J. (2000). A perception-action perspective on tool use development. Child Dev. 71, 137-144. doi: 10.1111/1467-8624.00127

Levine, S. C., Ratliff, K. R., Huttenlocher, J., and Cannon, J. (2011). Early puzzle play: a predictor of preschoolers' special transformation skill. Dev. Psychol. 48, 530-542. doi: 10.1037/a0025913

Marcinowski, E. C. (2015). How Does Handedness Affect the Development of Construction Skill From 10-24 Months? doctoral dissertation. University of North Carolina at Greensboro, Greensboro, NC.

Marcinowski, E. C., and Campbell, J. M. (2015). Building on what you have learned object construction skill during infancy predicts the comprehension of spatial relations words. Int. J. Behav. Dev. doi: 10.1177/0165025416635283

Michel, G. F. (1988). "A neuropsychological perspective on infant sensorimotor development," in Advances in Infancy Research, Vol. 5, eds C. K. Rovee-Collier and L. P. Lipsitt (Norwood, NJ: Ablex Pub. Corp), 1-37.

Michel, G. F. (1991). "Development of infant manual skills: motor programs, schemata, or dynamic systems," in The Development of Timing Control and Temporal Organization in Coordinated Action, eds J. Fagard and P. H. Wolff (Melville, NY: Elsevier), 175-199.

Michel, G. F. (1992). Maternal influences on infant hand-use during play with toys. Behav. Genet. 22, 163-176. doi: 10.1080/87565649809540723

Michel, G. F. (1998). A lateral bias in the neuropsychological functioning of human infants. Dev. Neuropsychol. 14, 445-469.

Michel, G. F. (2002). "Development of infant handedness", in Conceptions of Development: Lessons from the Laboratory, eds D. Lewkowicz and R. Lickliter (Philadelphia, PA: Psychology Press), 165-186.

Michel, G. F., Babik, I., Nelson, E. L., Campbell, J. M., and Marcinowski, E. C. (2013a). How the development of handedness could contribute to the development of language. Dev. Psychobiol. 55, 608-620. doi: 10.1037/a0033312

Michel, G. F., Babik, I., Sheu, C.-F., and Campbell, J. M. (2014). Latent classes in the developmental trajectories of infant handedness. Dev. Psychol. 50, 349-359. doi: $10.1002 / \mathrm{dev} .420190310$

Michel, G. F., and Harkins, D. A. (1986). Postural and lateral asymmetries in the ontogeny of handedness during infancy. Dev. Psychobiol. 19, 247-258.

Michel, G. F., Nelson, E. L., Babik, I., Campbell, J. M., and Marcinowski, E. C. (2013b). "Multiple trajectories in the developmental psychobiology of human handedness," in Embodiment and Epigenesis: Theoretical and Methodological Issues in Understanding the Role of Biology within the Relational Developmental System Part B: Ontogenetic Dimensions, eds R. M. Lerner and J. B. Benson (Melville, NY: Elsevier Inc), 227-260.

Nagin, D. (2005). Group-Based Modeling of Development. Cambridge, MA: Harvard University Press.

Nath, S., and Szucs, D. (2014). Construction play and cognitive skills associated with the development of mathematical abilities in 7-year-old children. Learn. Instruct. 32, 73-80. doi: 10.1016/j.infbeh.2013.01.009

Nelson, E. L., Campbell, J. M., and Michel, G. F. (2013). Unimanual to bimanual: tracking the development of handedness from 6 to 24 months. Infant Behav. Dev. 36, 181-188. doi: 10.1037/a0033803

Nelson, E. L., Campbell, J. M., and Michel, G. F. (2014). Early handedness in infancy predicts language ability in toddlers. Dev. Psychol. 50, 809-814. doi: 10.1037/11494-000

Piaget, J. (1952). The Origins of Intelligence in Childhood. New York, NY: International Universities Press.

Raudenbush, S., Bryk, A., Cheong, Y. F., Congdon, R., and du Toit, M. (2004). HLM 6: Hierarchical Linear \& Nonlinear Modeling. Lincolnwood, IL: Scientific Software International, Inc.

Vauclair, J. (1984). Phylogenetic approach to object manipulation in human and ape infants. Hum. Dev. 27, 321-328. doi: 10.1111/cdev.12165

Verdine, B. N., Golinkoff, R. K., Hirsh-Pasek, K., Newcombe, N. S., Filipowicz, A. T., and Change, A. (2014). Deconstructing building blocks: preschoolers spatial assembly performance relates to early mathematical skills. Child Dev. 85, 1062-1076. doi: $10.1177 / 0956797609354072$

Willems, R. M., Hagoort, P., and Casasanto, D. (2010). Body-specific representations of action verbs. Psychol. Sci. 21, 67-74. doi: $10.1080 / 0300443032000088212$ 
Wolfgang, C. H., Stannard, L. L., and Jones, I. (2003). Advanced constructional play with LEGOs among preschoolers as a predictor of later school achievement in mathematics. Early Child Dev. Care 173, 467-475. doi: $10.1080 / 0300443032000088212$

Conflict of Interest Statement: The authors declare that the research was conducted in the absence of any commercial or financial relationships that could be construed as a potential conflict of interest.
Copyright (C) 2016 Michel, Campbell, Marcinowski, Nelson and Babik. This is an open-access article distributed under the terms of the Creative Commons Attribution License (CC BY). The use, distribution or reproduction in other forums is permitted, provided the original author(s) or licensor are credited and that the original publication in this journal is cited, in accordance with accepted academic practice. No use, distribution or reproduction is permitted which does not comply with these terms. 